\title{
ASPECTOS EPIDEMIOLÓGICOS, CLÍNICOS E EVOLUTIVOS DA AIDS EM IDOSOS NO NORTE DO CEARÁ
}

EPIDEMIOLOGICAL, CLINICAL, AND EVOLUTIONARY ASPECTS OF AIDS IN THE ELDERLY IN

NORTHERN

CEARÁ

ASPECTOS EPIDEMIOLÓGICOS, CLÍNICOS Y EVOLUTIVOS DEL SIDA EN ANCIANOS EN EL NORTE

DE CEARÁ

Paulo Átila da Silva Viana ${ }^{1}$

Camila Teles Novais ${ }^{2}$

Rommel Wallace Costa Reis ${ }^{3}$

Sandra Maria Carneiro Flor 4

Patrícia Batista Rosa ${ }^{5}$

\section{RESUMO}

$E$

ste estudo teve por objetivo identificar os aspectos epidemiológicos, clínicos e evolutivos de idosos com síndrome da imunodeficiência adquirida (aids) atendidos em um centro de referência sorológico em Sobral (CE). Sua amostra foi composta por indivíduos com $\geq 59$ anos com caso notificado de aids no Centro de Orientação e Atendimento Sorológico (Coas), de janeiro de 2007 a dezembro de 2016. As informações foram coletadas em prontuários médicos e no Sistema Nacional de Agravos de Notificação (Sinan). Dos 72 prontuários selecionados, 61,11\% eram de indivíduos do gênero masculino, 54,17\% tinham entre $65 \mathrm{e}$ 74 anos, 31,94\% estudaram até o Ensino Fundamental, 70,8\% eram heterossexuais, 44,44\% relataram prática sexual desprotegida,

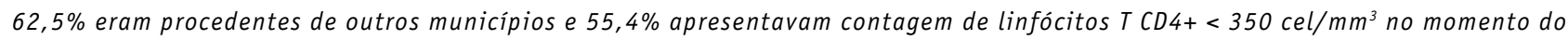
diagnóstico. Os principais sinais e sintomas que levaram os indivíduos a buscar o serviço de saúde, culminando com o diagnóstico, foram: perda de peso, diarreia, febre, leucoplasia pilosa e tosse persistente. Constatou-se a necessidade de intervenções voltadas à prevenção e à identificação de casos de doenças sexualmente transmissíveis (DST), para reduzir a incidência de casos de aids, tendo em vista sua elevada morbimortalidade na população idosa.

Palavras-chave: Sindrome da Imunodeficiência Adquirida; Epidemiologia; Idoso.

1. Aluno de graduação em Medicina Humana na Universidade Federal do Ceará (UFC). Membro da Liga de Infectologia de Sobral. Sobral (CE), Brasil.

2. Aluno de graduação em Medicina Humana na UFC. Membro da Liga de Infectologia de Sobral. Sobral (CE), Brasil.

3. Aluno de graduação em Medicina Humana na UFC. Membro da Liga de Infectologia de Sobral. Sobral (CE), Brasil.

4. Enfermeira. Especialista em Vigilância Epidemiológica e em Educação e Saúde para Preceptores do Sistema Único de Saúde (SUS) pelo Instituto Sírio Libanês de Ensino

e Pesquisa. Sobral (CE), Brasil.

5. Médica. Especialista em infectologia. Professora na UFC. Sobral (CE), Brasil. 


\section{ABSTRACT}

This study aimed to identify the epidemiological, clinical, and evolutionary aspects of the elderly with acquired immunodeficiency syndrome (AIDS) provided with care at a serological reference center in Sobral, Ceará, Brazil. Its sample consisted of individuals aged $\geq 59$ years with a case of AIDS notified to the Brazilian Serological Advise and Care Center (COAS), from January 2007 to December 2016. Information was collected both from medical records and the Brazilian Information System for Notifiable Diseases (SINAN). Out of the 72 medical records selected, $61.11 \%$ belonged to men, $54.17 \%$ were between 65 and 74 years old, $31.94 \%$ have studied up to Elementary School, $70.8 \%$ were heterosexual, $44.44 \%$ reported unprotected sexual intercourse, $62.5 \%$ were from other municipalities, and $55.4 \%$ had a CD4+T-cell count $<350$ cells $/ \mathrm{mm}^{3}$ at the time of diagnosis. The main signs and symptoms that led individuals to seek the health service, culminating in the diagnosis, were: weight loss, diarrhea, fever, hairy leukoplakia, and persistent cough. The need for interventions aimed at prevention and identification of cases of sexually transmitted diseases (STD) was found, in order to reduce the incidence of AIDS cases, due to their high morbidity and mortality rates in the elderly.

Keywords: Acquired Immunodeficiency Syndrome; Epidemiology; Elderly.

\section{RESUMEN}

Este estudio tuvo como objetivo identificar los aspectos epidemiológicos, clínicos y evolutivos de ancianos con síndrome de inmunodeficiencia adquirida (SIDA) atendidos en un centro de referencia serológico en Sobral, Ceará, Brasil. Su muestra consistió en individuos de $\geq 59$ años con un caso de SIDA notificado al Centro de Orientación y Atención Sorológico Brasileño (COAS), de enero de 2007 a diciembre de 2016. La información se recopiló tanto de registros médicos como del Sistema Nacional de Agravios de Notificación Brasileño (SINAN). De los 72 registros médicos seleccionados, $61,11 \%$ pertenecian a hombres, 54,17\% tenían entre 65 y 74 años, 31,94\% habían estudiado hasta la Enseñanza Fundamental, 70,8\% eran heterosexuales, 44,44\% reportaron relaciones sexuales sin protección, 62,5\% eran de otros municipios y 55,4\% tenían un recuento de células T CD4+ < 350 células/ $\mathrm{mm}^{3}$ en el momento del diagnóstico. Los principales signos y síntomas que llevaron a las personas a buscar el servicio de salud, que culminaron en el diagnóstico, fueron: pérdida de peso, diarrea, fiebre, leucoplasia vellosa y tos persistente. Se encontró la necesidad de intervenciones dirigidas a la prevención y la identificación de casos de enfermedades sexualmente transmisibles (EST), para reducir la incidencia de casos de SIDA, debido a sus altas tasas de morbilidad y mortalidad en los ancianos.

Palabras clave: Sindrome de Inmunodeficiencia Adquirida; Epidemiología; Anciano.

\section{INTRODUÇÃO}

0 combate à síndrome da imunodeficiência adquirida (aids) tem sido árduo e obstinado, trazendo ganhos significativos no que diz respeito à morbimortalidade nos últimos anos, principalmente em termos de sua prevenção e seu tratamento. Metas têm sido estabelecidas em escala global entre os diversos países, com o intuito de eliminar e mitigar novas infecções pelo vírus da imunodeficiência humana (HIV) ${ }^{1}$.

Mais de 3 décadas se passaram desde sua descoberta e na última delas se observou o início da estabilização da incidência de infecção pelo HIV no Brasil, apesar do significativo crescimento linear do número de casos nas regiões Norte e Nordeste do país. A taxa de detecção da aids apresenta média nacional de 20,5 casos para cada 100 mil habitantes, contra 11,7 casos para cada 100 mil habitantes no Nordeste, região que se encontra na terceira posição em termos do número de óbitos identificados que tiveram essa síndrome como causa base, atrás do Sudeste e do Sul no ranking, respectivamente. Além disso, nos últimos 10 anos houve aumento de $34,3 \%$ da mortalidade no Nordeste, contrapondo-se à queda da incidência nacional no mesmo período ${ }^{2}$.

No Ceará, foram notificados 16.790 casos de aids de 1983 até 2016, com gradativo aumento até 2012 e posterior declínio. Em 2016 foram notificados 202 casos nesse estado, representando a menor taxa de detecção da série histórica, o que pode ser justificado pelo aumento do diagnóstico precoce da infecção por HIV. Entre 2007 e 2016 identificou-se o maior número de casos de aids no Ceará, concentrando-se no sexo masculino, embora 23 municípios tenham alcançado maior número entre as mulheres, sugerindo o início da feminização dessa doença ${ }^{3}$.

A faixa etária dos idosos, nos últimos anos, tem constituído foco de preocupação quanto ao aumento da incidência de casos de HIV/aids. Estudos focados na investigação do comportamento sexual em indivíduos idosos apontam menor conscientização quanto aos fatores de risco e estratégias de prevenção. Um estudo de séries históricas 
no Ceará, no período de 2001 a 2011, verificou que a faixa etária dos idosos teve pico de detecção da aids em 2010 (4,2 casos para cada 100 mil habitantes), sendo esse valor quase 5 vezes maior do que o de 2001, que teve 0,9 para cada 100 mil habitantes. Dentre os municípios com maior crescimento de casos no estado, Sobral destacou-se por apresentar aumento significativo entre 2004 e $2009^{4,5}$.

Diante da progressiva tendência de aumento do número de casos de aids em idosos no Ceará e do aumento da expectativa de vida dos infectados por HIV, em vista da evolução do diagnóstico e do tratamento, mostra-se necessário avaliar os aspectos epidemiológicos, clínicos e evolutivos da aids em idosos dos diferentes municípios no estado, para adotar ações localizadas e específicas voltadas à sexualidade dos idosos para a prevenção de doenças.

\section{METODOLOGIA}

Trata-se de estudo epidemiológico e descritivo, por meio de fontes secundárias de dados, realizado no Centro de Orientação e Atendimento Sorológico (Coas) em Sobral. A coleta de dados foi feita a partir de prontuários médicos e do Sistema Nacional de Agravos de Notificação (Sinan). 0 Coas constitui referência para o atendimento de pacientes com HIV/aids e pertence à $11^{\mathrm{a}}$ Coordenadoria Regional de Saúde (CRES), que compõe a macrorregião de Sobral, abrangendo aproximadamente 630 mil habitantes dentre 24 municípios: Alcântaras, Cariré, Catunda, Coreaú, Forquilha, Frecheirinha, Graça, Groaíras, Hidrolândia, Ipu, Irauçuba, Massapê, Meruoca, Moraújo, Mucambo, Pacujá, Pires Ferreira, Reriutaba, Santa Quitéria, Santana do Acaraú, Senador Sá, Sobral, Uruoca e Varjota.

Os critérios de inclusão foram: idade $\geq 59$ anos; caso notificado de aids entre $1^{\circ}$ de janeiro de 2007 e 31 de dezembro de 2016; tratamento iniciado com antirretroviral na instituição. Os critérios de exclusão foram: prontuários incompletos; e transferência de pacientes para tratamento em outro centro de referência.

As variáveis de interesse da análise foram os dados sociodemográficos, clínicos e laboratoriais: sexo, idade, escolaridade, ocupação, procedência, contagem de células CD4+ e carga viral. 0 diagnóstico da aids se baseou no Critério Rio de Janeiro/Caracas, fundamentado na existência de 2 testes de triagem reagentes ou 1 teste confirmatório para detecção de anticorpos anti-HIV, além do somatório de pelo menos 10 pontos, de acordo com uma escala de sinais, sintomas ou doenças ${ }^{6}$.

As informações foram analisadas em termos estatísticos, por meio do programa computacional Epi Info, versão 7.0, com aplicação do teste $t$ de Student para variáveis não pareadas e teste de Fischer, visando a identificar significância

\section{...o número de homens infectados com aids supera \\ o número de mulheres...}

estatística entre as categorias de variáveis. Admitiu-se valor de $p<0,05$.

Este estudo foi aprovado pelo Comitê de Ética em Pesquisa da Universidade Federal do Ceará (UFC), sob o Protocolo n. 3.272/2017. 0 termo de consentimento livre e esclarecido não foi aplicado, por se tratar de pesquisa com dados secundários. Todos os preceitos éticos pertinentes foram contemplados, de acordo com a Resolução do Conselho Nacional de Saúde (CNS) n. 466/2012.

\section{RESULTADOS E DISCUSSÃO}

A taxa de detecção absoluta da aids entre 2007 e 2016 foi de 803 casos, dos quais $8,96 \%$ atenderam aos critérios desta pesquisa. Quanto ao sexo, notou-se que o número de homens infectados com aids supera o número de mulheres $(61,11 \%)$. Entretanto, analisando a distribuição dos casos ao longo do período de estudo, constatou-se o surgimento da feminização dessa doença.

Essa tendência encontra possiveis justificativas na maior vulnerabilidade feminina, na limitação de acesso aos serviços de saúde reprodutiva, além de dificuldades na negociação do uso de preservativo com o parceiro ${ }^{7}$. Além disso, as alterações anatomofisiológicas do processo natural de envelhecimento entre as mulheres, tais como a redução do canal vaginal e o enfraquecimento de suas paredes, bem como a diminuição da elasticidade e das secreções vaginais, corroboram a maior susceptibilidade feminina ao risco da infecção por HIV em relações sexuais - fato contrário ao senso comum ${ }^{8}$.

Em relação aos aspectos sociodemográficos analisados neste estudo, evidenciou-se que o grupo etário mais acometido na população idosa foi dos 65 aos 74 anos $(54,17 \%)$, seguido pelo grupo dos 58 aos 64 anos $(45,83 \%)$. A Tabela 1 também ilustra a distribuição dos casos de aids em idosos por município de procedência, com destaque para Sobral (37,5\% dos casos no período avaliado).

Tais evidências corroboram um estudo realizado em Pernambuco, em 2007, indicando que a faixa etária dos 50 aos 59 anos teve a segunda maior incidência de aids por idade no país; a partir de 1995, a incidência propagou-se na faixa etária dos 60 aos 69 anos. Contata-se o crescimento do 
número de casos na população idosa9 ${ }^{9}$.

Tabela 1 - Dados sociodemográficos. Sobral, 2007-2016.

\begin{tabular}{lcc}
\hline Variáveis & $\mathbf{N}=\mathbf{7 2}$ & $(\%)$ \\
\hline Sexo & 44 & 61,1 \\
Masculino & 28 & 38,8 \\
Feminino & & \\
Faixa etária & 33 & 45,8 \\
58 a 64 anos & 39 & 54,2 \\
65 a 74 anos & & \\
Escolaridade & 13 & 17,6 \\
Alfabetizado & 21 & 28,4 \\
Analfabeto & 8 & 12,2 \\
Ignorado & 23 & 31,1 \\
Ensino Fundamental & 6 & 9,5 \\
Ensino Médio & 1 & 1,4 \\
Ensino Superior completo & & \\
Procedência & 27 & 37,5 \\
\hline Sobral & 45 & 62,5 \\
Outras cidades do Ceará & & \\
\hline Fonte:Elaborado & & \\
\hline
\end{tabular}

Fonte: Elaborado pelos autores.

Quanto à escolaridade dos idosos, a grande maioria tinha Ensino Fundamental ou era analfabeta - 31,94\% e $28,38 \%$, respectivamente. Entretanto, foi identificado 1 indivíduo com Ensino Superior no ano de 2010. 0 grau de escolaridade constitui importante indicador do nível socioeconômico, assim, tem grande impacto sobre a transmissão de doenças sexualmente transmissíveis (DST). Tal fato justifica-se pela maior suscetibilidade desse grupo, por falta de compreensão dos riscos e métodos preventivos e pela pior cobertura dos sistemas de vigilância e de assistência à saúde ${ }^{10}$.

Quanto à procedência, observou-se um processo de interiorização da aids, com a maioria dos casos $(62,5 \%)$ oriundos de regiões distantes da cidade polo. Isso sugere que, além do processo de interiorização, com índices crescentes de infecção no interior do estado, existe falha no tratamento e na assistência das complicações desses novos infectados na faixa etária de estudo. Fato este que corrobora diversos estudos sobre essa têndencia no país - como, por exemplo, em Minas Gerais ${ }^{11}$.

Quanto à categoria de exposição, 23,6\% não continham essa informação descrita em todos os prontuários, provavelmente por erro na coleta de dados ou no preenchimento da notificação, mas $70,8 \%$ dos idosos afirmaram ser heterossexuais, 2,8\% eram bissexuais e $2,8 \%$ eram homossexuais. No que diz respeito ao uso de preservativo, $47,22 \%$ dos prontuários também não continham essa informação, porém, uma grande parcela $(44,44 \%)$ declarou não fazer uso e somente $6,94 \%$ relataram uso contínuo (Tabela 2).

34 - SANARE, Sobral - V.16 n.02,p.31-36, Jul./Dez. - 2017
Tabela 2 - Categoria de exposição ao HIV e uso de métodos preventivos. Sobral, 2007-2016.

\begin{tabular}{lccccc}
\hline Variáveis & Masc. & $(\%)$ & Fem. & $(\%)$ & Total \\
\hline Categoria de exposição & & & & \\
Bissexual & 2 & 2,8 & 0 & 0,0 & $2,8 \%$ \\
Heterossexual & 30 & 41,6 & 21 & 29,1 & $70,7 \%$ \\
Homossexual & 2 & 2,8 & 0 & 0 & $2,8 \%$ \\
Ignorado & 10 & 13,9 & 7 & 16,6 & $30,5 \%$ \\
Uso de preservativo & & & & \\
Usava & 5 & 7,0 & 0 & 0,0 & $7,0 \%$ \\
Não usava & 18 & 25,0 & 14 & 19,4 & $44,4 \%$ \\
Ignorado & 12 & 16,7 & 8 & 11,11 & $27,8 \%$ \\
\hline
\end{tabular}

Fonte: Elaborado pelos autores.

Nos idosos, a transmissão do HIV ocorre principalmente no grupo de comportamento heterossexual, isso porque são observadas mudanças no comportamento sexual de homens e mulheres da terceira idade em termos de sexo seguro. Umas das explicações para a baixa adesão é a falta de hábito, pois não constituía prática padrão na formação cultural e os homens temem a perda de ereção durante a relação sexual. Além disso, nessa faixa etária a população feminina, por apresentar menor probabilidade de gravidez, enfrenta maiores dificuldades de negociação do uso de preservativo com seus parceiros ou simplesmente opta pelo sexo desprotegido por questões de comodidade ${ }^{12,13}$.

Dentre os motivos que levaram os idosos a procurar os serviços de saúde e que resultaram no diagnóstico de aids, os mais frequentes foram: caquexia ou perda de peso $>10 \%$ $(60,81 \%)$, diarreia $\geq 1$ mês $(58,11 \%)$, febre $\geq 38^{\circ}$ por $>1$ mês $(56,76 \%)$, astenia $>1$ mês $(48,65 \%)$, tosse persistente ou qualquer pneumonia $(37,84 \%)$, candidose oral ou leucoplasia pilosa $(28,38 \%)$ e dermatite persistente $(27 \%)$. Os sinais e sintomas foram distribuídos de acordo com o critério Rio de Janeiro/Caracas, para facilitar sua identificação (Tabela 3 ).

Tabela 3 - Sinais/sintomas que levaram os idosos aos serviços de saúde. Sobral, 2007-2016.

\begin{tabular}{lcc}
\hline Variáveis & N = 72 & (\%) \\
\hline Perda de peso $>10 \%$ & 44 & 60,8 \\
Diarreia $\geq 1$ mês & 41 & 58,1 \\
\hline Febre $\geq 38^{\circ}$ por $\geq 1$ mês & 42 & 56,8 \\
Astenia $\geq 1$ mês & 36 & 48,7 \\
\hline Tosse persistente ou qualquer pneumonia & 28 & 37,8 \\
Candidose oral ou leucoplasia pilosa & 21 & 28,4 \\
\hline Dermatite persistente & 20 & 27,0 \\
\hline
\end{tabular}

Fonte: Elaborado pelos autores.

Os idosos, em virtude dos agravos decorrentes da maior fragilidade fisiológica inerente ao processo de envelhecimento, associados à redução da defesa imunológica causada pela doença, apresentam maior tendência a adoecer 
e, consequentemente, evoluir a óbito com maior rapidez. Em muitos casos, o vagaroso diagnóstico da doença, combinado a outras enfermidades, potencializa e acelera a progressão da síndrome, culminando com a morte, a qual, não raramente, é declarada por outras causas, implicando na subnotificação dessa categoria ${ }^{14}$.

A letalidade da aids atingiu sobremaneira o gênero masculino, sendo constatadas, nesse grupo, $66,6 \%$ das mortes no período do estudo - com pico em 2016. 0 desfecho morte vem apresentando crescimento linear, como se observa neste estudo - que revela aumento do número de óbitos no último ano, passando de 1 caso em 2015 para 3 casos em 2016 (Tabela 4).

Tabela 4 - Casos de óbito por aids em indivíduos com $\geq 59$ anos. Sobral, 2007-2016.

\begin{tabular}{lccccccccccccc}
\hline \multirow{2}{*}{ Sexo } & \multicolumn{1}{c}{ Número de óbitos por ano } & \multicolumn{1}{c}{ Total } \\
\cline { 2 - 13 } & 2007 & $\mathbf{2 0 0 8}$ & $\mathbf{2 0 0 9}$ & $\mathbf{2 0 1 0}$ & $\mathbf{2 0 1 1}$ & $\mathbf{2 0 1 2}$ & $\mathbf{2 0 1 3}$ & $\mathbf{2 0 1 4}$ & $\mathbf{2 0 1 5}$ & $\mathbf{2 0 1 6}$ & $\mathbf{N}$ & $\%$ \\
\hline Masculino & 1 & 1 & 0 & 1 & 0 & 0 & 0 & 3 & 0 & 2 & 8,0 & $66,7 \%$ \\
Feminino & 0 & 1 & 1 & 0 & 0 & 0 & 0 & 0 & 1 & 1 & 4,0 & $33,3 \%$ \\
Total & 1 & 2 & 1 & 1 & 0 & 0 & 0 & 3 & 1 & 3 & 12 & $100,0 \%$ \\
\hline
\end{tabular}

Fonte: Elaborado pelos autores.

Em relação à letalidade, estudos demonstram em diversos países uma significativa tendência de queda. Embora nos primeiros anos a epidemia alcance $100 \%$ de óbitos, desde 1996 o perfil de mortalidade por aids decresce nos países ricos e cresce nos países pobres, totalizando, em termos gerais, uma diminuição de $50 \%$ dos casos com morte. 0 Brasil se encontra entre os países com redução da mortalidade por aids - cujo início coincide com a adoção da política de distribuição gratuita de antirretrovirais aos portadores de HIV ${ }^{15}$.

No que diz respeito aos níveis de linfócitos CD4+ no momento do diagnóstico, 55,4\% dos idosos apresentavam contagem de linfócitos T CD4+ $350 \mathrm{cel} / \mathrm{mm}^{3}$ na investigação diagnóstica. Foi correlacionada a variável níveis de linfócitos CD4+ à evolução do quadro clínico, apresentando tendência significativa para preditor de mortalidade os indivíduos com contagem de linfócitos $\mathrm{T}$ CD4+ $<350 \mathrm{cel} / \mathrm{mm}^{3}$ no momento do diagnóstico de aids ( $\left.\mathrm{RR}=5,31, \mathrm{IC} 95 \%=1,19-23,68, p=0,012\right)$, como ilustra a Tabela 5.

Tabela 5 - Correlação entre os níveis de linfócitos T CD4+ e morte entre idosos com aids. Sobral, 2007-2016.

\begin{tabular}{lccccc}
\hline Variáveis & Desfecho & N $(\%)$ & RR & IC 95\% & P \\
\hline Níveis de linfócitos T CD4+ $<$ & Vivos & $39(54,16)$ & 5,31 & $1,19-23,68$ & 0,012 \\
$350 \mathrm{cel} / \mathrm{mm}^{3}$ & Mortos & $2(2,77)$ & & & \\
\hline
\end{tabular}

Fonte: Elaborado pelos autores.

Tais dados comprovam a relação entre diagnóstico tardio e prognóstico reservado, uma vez que os indivíduos só procuram atendimento quando apresentam sinais e sintomas característicos da infecção ou devido a enfermidades oportunistas, isto é, quando a doença já se encontra em fase mais avançada e com repercussões de difícil controle ou desfecho morte ${ }^{16}$.

\section{CONSIDERAÇÕES FINAIS}

Este estudo promoveu um esquadrinhamento dos aspectos epidemiológicos, clínicos e evolutivos dos idosos com aids atendidos no Coas de Sobral, mediante levantamento de informações de prontuários e do Sinan. Como dificuldades, citamos as lacunas de informações decorrentes de prontuários mal preenchidos. Apesar disso, acredita-se que esta pesquisa contribui com o desenvolvimento de ações de prevenção e rastreio da aids nessa população.

$0 s$ resultados levantados neste estudo demonstram que a prevalência se deu em pacientes do gênero masculino, com idade entre 65 e 74 anos, com Ensino Fundamental completo, heterossexuais, com prática sexual desprotegida, procedentes de localidades fora de Sobral e com contagem de linfócitos $T$ $\mathrm{CD} 4+<350 \mathrm{cel} / \mathrm{mm}^{3}$ no momento do diagnóstico. Além disso, na correlação de variáveis, evidenciou-se significância do preditor de mortalidade e níveis de linfócitos T CD $4+<350$ $\mathrm{cel} / \mathrm{mm}^{3}$ no momento da notificação.

Diante desse cenário, mostram-se necessárias intervenções voltadas à prevenção e ao diagnóstico precoce de DST na população idosa, para que diminua a incidência e os prognósticos reservados dos casos de aids. Tais intervenções devem ser direcionadas - sobretudo para a utilização de métodos de preventivos, como o uso de preservativos nas relações sexuais - e coordenadas pela atenção primária à saúde (APS), por conta de sua proximidade com a comunidade. Para tanto, há necessidade de capacitação dos profissionais da saúde e de constante debate com a comunidade acerca da temática, tendo em vista o preconceito quanto à sexualidade da população idosa.

SANARE, Sobral - V.16 n.02,p.31-36, Jul./Dez. - 2017 - 35 


\section{CONTRIBUIÇÃO DOS AUTORES}

Paulo Átila da Silva Viana contribuiu com o delineamento da pesquisa, a coleta e análise de dados e a redação do manuscrito. Camila Teles Novais contribuiu com o delineamento da pesquisa, a revisão de literatura e a redação do manuscrito. Rommel Wallace Costa Reis contribuiu com o delineamento da pesquisa, a coleta de dados e a redação do manuscrito. Sandra Maria Carneiro Flor contribuiu com a coleta de dados. Patrícia Batista Rosa contribuiu com a revisão crítica do manuscrito.

\section{REFERÊNCIAS}

1. Joint United Nations Programme on HIV/Aids. Global AIDS response progress reporting 2016: construction of core indicators for monitoring the 2011 UN Political Declaration on HIV/AIDS. Genebra: WHO; 2016.

2. Brasil, Boletim epidemiológico: Aids e DST - $1^{\text {a }}$ a $26^{\text {a }}$ semanas epidemiológicas. 2015; 4(1).

3. Ceará (Estado). Informe Epidemiológico HIV/Aids. Fortaleza: Secretaria de Saúde; 2016.

4. Pedrosa NL, Paiva SS, Almeida RL, Holanda ER, Kerr LR, Galvão MT.. The historic data series on AIDS in the State of Ceará, Brazil. Ciênc Saúde Coletiva [serial on the internet]. 2015 [cited 2017 Nov 24];20(4):1177-84, Available from: http://www.scielo.br/pdf/csc/v20n4/1413-8123csc-20-04-01177.pdf

5. Lima GK, Mesquita K0, Flôr SMC, Freitas CASL, Linhares MSC. Evolução historica da aids no municipio de Sobral, Ceará, Brasil, no período de 2004 a 2010. Sanare (Sobral, Online) [serial on the internet]. 2011 [cited 2017 Nov 24];10(2):50-6. Available from: file:///D:/255-487-1-SM.pdf

6. Brasil. Programa Nacional de DST e aids. Brasília (DF): Ministério da Saúde; 2004. (Série Manual, n. 60).

7. Castilho EA, Rodrigues-Junior AL. A epidemia da aids no Brasil, 1991-2000. Rev Bras Soc Med Trop [serial on the internet]. 2004 [cited 2017 Nov 24];37(4):312-7. Available from: https://social.stoa. usp.br/articles/0033/9645/2004_A_epidemia_de_AIDS no Brasil 1991-2000-descri\%C3\%A7\%C3\%A30 espa\%C3\%A7o-temporal.pdf

8. Araújo VLB, Brito DMS, Gimeniz MT, Queiroz TA, Tavares CM. Características da aids na terceira idade em um hospital de referência do Estado do Ceará, Brasil. Rev Bras Epidemiol [serial on the internet]. 2007 [cited 2017 Nov 24];10(4):544-54. Available from: http://www.scielo.br/ $\mathrm{pdf} / \mathrm{rbepid} / \mathrm{v} 10 \mathrm{n} 4 / 12 . \mathrm{pdf}$

9. Pottes FA, Brito AM, Gouveia GC, Araújo EC, Carneiro RM. Aids e envelhecimento: características dos casos com idade igual ou maior que 50 anos em Pernambuco, de 1999 a 2000.
Rev Bras Epidemiol [serial on the internet]. 2007 [cited 2017 Nov 24];10(3):338-51. Available from: http://www.scielo. $\mathrm{br} / \mathrm{pdf} / \mathrm{rbepid} / \mathrm{v} 10 \mathrm{n} 3 / 04 . \mathrm{pdf}$

10. Driemeier M, Andrade SMOD, Pontes ERJC, Paniago AMM, Cunha RVD. Vulnerability to AIDS among the elderly in an urban center in Central Brazil. Clinics [serial on the internet]. 2012 [cited 2017 Nov 24];67(1):19-25. Available from: http://www.scielo.br/pdf/clin/v67n1/v67n1a04.pdf

11. Reis CT. A interiorização da epidemia de HIV/aids e o fluxo intermunicipal de internação hospitalar na Zona da Mata, Minas Gerais, Brasil: uma análise espacial. Cad Saúde Pública [serial on the internet]. 2008 [cited 2017 Nov 24];24(6):1219-28. Available from: http://www.scielo.br/ $\mathrm{pdf} / \mathrm{csp} / \mathrm{v} 24 \mathrm{n} 6 / 03 . \mathrm{pdf}$

12. Eucebious LM. Exploring gender perceptions of risk of HIV infection and related behaviour among elderly men and women of Ga-Rankuwa, Gauteng Province, South Africa. SAHARA-J: Journal of Social Aspects of HIV/AIDS [serial on the internet]. 2016 [cited 2017 Nov 24];13(1):88-95. Available from: http://www.tandfonline.com/doi/full/10.10 $\underline{80 / 17290376.2016 .1218790}$

13. Driemeier M, Andrade SMOD, Pontes ERJC, Paniago AMM, Cunha RVD. Vulnerability to AIDS among the elderly in an urban center in central Brazil. Clinics [serial on the internet]. 2012 [cited 2017 Nov 24];67(1):19-25. Available from: http://www.scielo.br/pdf/clin/v67n1/v67n1a04.pdf

14. Santos NJS, Tayra A, Silva SR, Buchalla CM, Laurenti R. A aids no estado de São Paulo: as mudanças no perfil da epidemia e perspectivas da vigilância epidemiológica. Rev Bras Epidemiol [serial on the internet]. 2002 [cited 2017 Nov 24];5(3):286-310. Available from: http://www.scielo. br/pdf/rbepid/v5n3/07.pdf

15. Fonseca M, Barreira DA. A evolução da mortalidade por aids no país segundo sua distribuição geográfica. Bol Epidemiol DST/AIDS. 2000;13(3).

16. Ultramari L, Moretto PB, Gir E, Canini SRMS, Teles SA, Gaspar J, Machado AA. Perfil clínico e epidemiológico da infecção pelo HIV/aids em idosos. Rev Eletrônica Enferm [serial on the internet]. 2011 [cited 2017 Nov 24];13(3):40512. Available from: https://www.fen.ufg.br/fen_revista/ $\mathrm{v} 13 / \mathrm{n3} / \mathrm{pdf} / \mathrm{v} 13 \mathrm{n} 3 \mathrm{a} 05 . \mathrm{pdf}$

Recebido em 05/06/2017 Aprovado em 09/11/2017
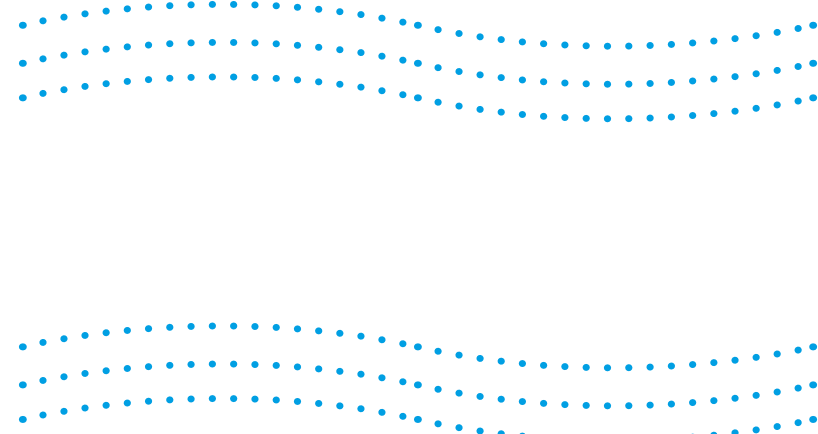